\title{
IoT-Based Power Security and Prepaid Electricity System
}

\author{
Sakshi Patil ${ }^{1}$, Aaksha Jaywant ${ }^{2}$ \\ T.E., Electronics and Telecommunication Engineering, VES Institute of Technology, Mumbai-400074, India ${ }^{1}$
}

T.E., Electronics \& Telecommunication Engineering, Bharati Vidyapeeth College of Engineering, Navi Mumbai, India ${ }^{2}$

\begin{abstract}
This paper presents the advanced IoT based concept wherein electricity bills are switched to prepaid facility. This work is used to monitor power consumption and automatically alerts the user to recharge the account through the Internet and SMS. An Arduino processor is used to monitor and control entire system model and the GSM Technology is used to update the user about the account through the SMS service. This is for people in the rural areas where internet has still not managed to create its hold on a daily basis. The IoT based system is used to update the data whereas the APIs are used to notify tech savvy people via emails and notifications. This process is periodic in nature and hence power reduction takes place at a greater rate. This technology finds application in electricity distribution companies, corporates, household and rural areas where power theft is still an issue. The implementation of this project will result in better energy management, conservation of energy and eradicate unnecessary hassle over incorrect billing. The automated billing system keeps track of time and energy consumption simultaneously leaving little scope for disagreement on consumption and billing. This project provides major benefits of data collection that allow substantial saving through reduction of meter re-read, greater data accuracy, frequent reading and improved customer service
\end{abstract}

Keywords: Prepaid electricity, Power security, Arduino controller, GSM/GPRS, IoT, APIs.

\section{INTRODUCTION}

The Existing Automatic Meter Reading System uses AT89S52 microcontroller which consumes more power. The proposed method provides the communication between the Electricity Board section and the consumer section using Internet of Things (IOT) for transmitting the customer's electricity consumption and bill information that is calculated using advanced Arduino microcontroller unit. The system uses advanced microcontroller Arduino unit that continuously monitors and records the Energy Meter readings in its permanent (non-volatile) memory location. It also makes use of a GSM modem for monitoring, reading and controlling of Energy Meter from a remote server automatically using the existing GSM networks for cellular phones. The GSM infrastructure, has nationwide coverage, which can be used to request and retrieve power consumption notification over individual houses and flats. The power fluctuations are monitored using the voltage sensor and current sensor are fed to the Arduino which indicates it to the Electricity Board. Depending on the power generation, the house hold devices are controlled automatically. From Electricity Board section the information regarding the bill amount and payment are communicated to the consumer via Global System for Mobile communication. The power and billing information is continuously transmitted by the use of Internet of Things and monitored by the Electricity Board section.

\section{PROPOSED SYSTEM}

IoT is the latest system which allows monitoring of energy consumption at lower costs as the complete data is sent over internet to a central server. Electricity is made available only when the account is recharged which allows the saving of power. Android users can pay their electric bills from their android application where as non-android users can check their usage and make payments online. The system is more reliable and accurate reading values are collected from energy meters. As it allows easy monitoring and billing facility, it lessens work load and thus saves time. The data is stored on a central server and is easily accessible for many. The communication medium is secure and tampering of energy meters can be identified easily. If an error occurs in the system, the value in the central server will not be updated. Once the value updated crosses the threshold time, the server can determine that something is wrong in the system and can report to the engineers. Thus, identification of error becomes easier. Thus, identification of error becomes easier. Since the values are stored in the central database, the reports are made accessible from anywhere in the world. Also, the server is online $24 \times 7$.

III. SYSTEM DESIGN

It is sub-divided into 2 units- Hardware system design and Web-sever design. The hardware system design consists of components in the circuit diagram and pulse calculation of the energy units. The web-server design consists of AT commands given to the GSM and Arduino programming done in order to connect to the Central server using IoT. 


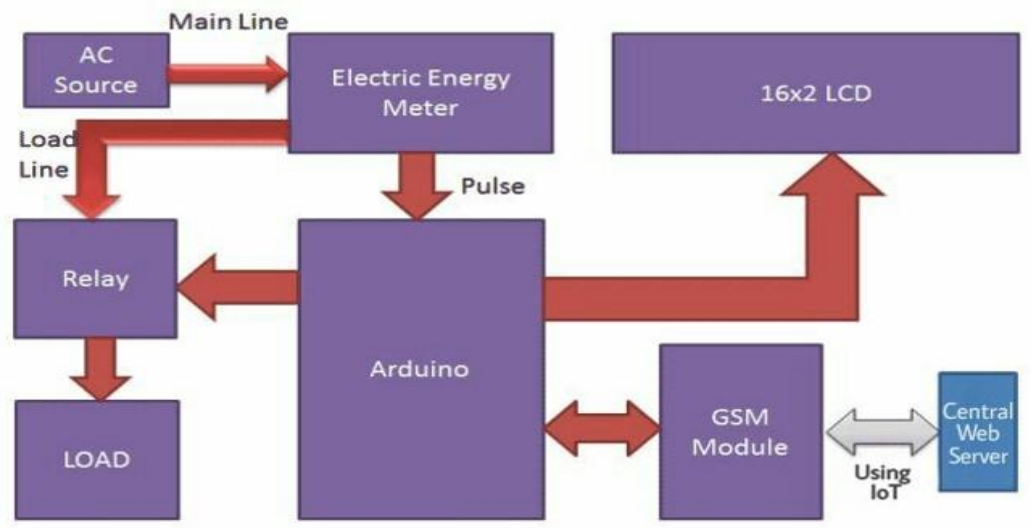

A. Hardware System Design

Fig. 1 System Design

\section{ENERGY SYSTEM}

An energy meter is a device that measures the amount of electric energy consumed by a residence, a business or an electrically powered.

The conventional mechanical energy meter is based on the phenomenon of "Magnetic Induction". It has a rotating aluminum Wheel called Ferriwheel and many toothed wheels. Since many mechanical parts are involved, mechanical defects and breakdown are common. More over chances of manipulation and current theft will be higher.

Electronic Energy Meter (EEM) known as the "Static Energy Meter" is based on Digital Micro Technology (DMT) and uses no moving parts. In EEM the accurate functioning is controlled by a specially designed IC called ASIC (Application Specified Integrated Circuit). ASIC is constructed only for specific applications like Washing Machines, Air conditioners, Automobiles etc. using Embedded System Technology. In addition to ASIC, EEM also consists of analogue circuits, Voltage transformer, Current transformer to "Sample" current and voltage. Usually 800 to 3600 pulses / $\mathrm{kWh}$ generating ASICs are used in EEMs.

\section{ARDUINO CONTROLLER}

The Uno is a microcontroller board based on the ATmega328P. The Atmega328 is a very popular microcontroller chip produced by Atmel. It is an 8-bit microcontroller that has $32 \mathrm{~K}$ of flash memory, $1 \mathrm{~K}$ of EEPROM, and $2 \mathrm{~K}$ of internal SRAM. Atmega 328 has $32 \mathrm{~K}$ of flash program memory and $2 \mathrm{~K}$ of Internal SRAM. The Atmega328 has 28 pins. It has 14 digital I/O pins, of which 6 can be used as PWM outputs and 6 analog input pins. These I/O pins account for 20 of the pins. Arduino digital pin number 7, 6, 5, 4, 3, 2 are connected to the Data pins of LCD namely RS, EN, D4, D5, D6, D7. Also, Tx and Rx pins of Arduino are connected to the Rx and Tx pins of GSM module respectively. Electricity energy meter is interfaced with Arduino using the pulse LED (Calibration or Cal) of electricity Energy meter. We only need to connect this CAL LED to Arduino through an Optocoupler IC. A relay is used for switching electricity connection which is connected at pin 12 of Arduino though ULN2003 relay driver.

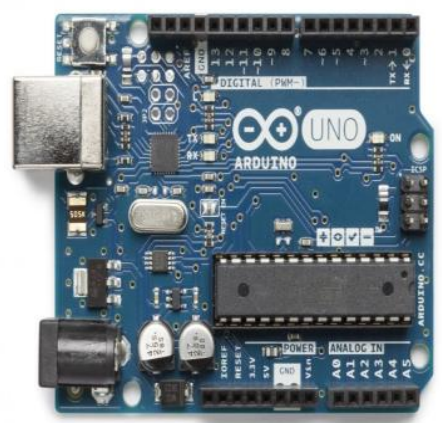

Fig. 2 Arduino Controller Atmega328P

\section{GSM SIM 900}

The SIM900 is a complete Quad-band GSM/GPRS solution in a SMT module which can be embedded in the customer applications .GSM/GPRS Modem-RS232 is built with Quad Band GSM/GPRS engine- SIM900, works on frequencies 850/ 900/ 1800/ $1900 \mathrm{MHz}$ The Modem is coming with RS232 interface which allows you connect PC as well as microcontroller with RS232 Chip (MAX232). The baud rate is configurable from 9600-115200 (default baud rate is 9600) through AT command. The GSM/GPRS Modem is having internal TCP/IP stack to enable you to connect with internet via GPRS. With a tiny configuration of $24 \mathrm{~mm} \times 24 \mathrm{~mm} \times 3 \mathrm{~mm}$, SIM900 can fit almost all the space requirements in your M2M application, especially for slim and compact demand of design. 


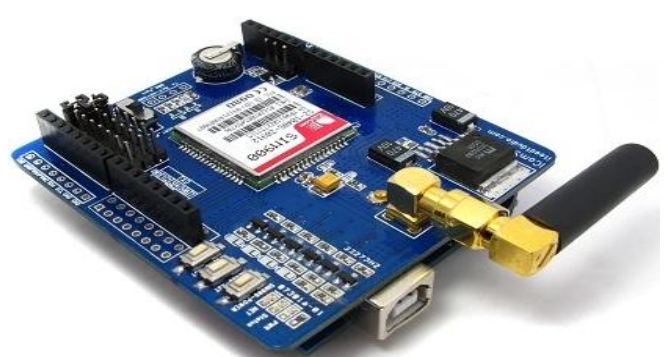

Fig. 3 GSM SIM 900

\section{OPTOCOUPLER}

4N35 is an optocoupler integrated circuit in which an infrared emitter diode drives a phototransistor. They are also known as optoisolators since they separate two circuits optically. These are used to couple two circuits without any ohmic contact. They allow one of the circuits to switch another one while they are completely separate. The first circuit is connected to IR diode while the other circuit with the phototransistor. The isolation ensures that no damage occurs in either of the circuits while the other one has a fault.

An optocoupler is analogous to a relay which isolates two circuits magnetically. They differ with relays in the sense that they are smaller in size and allow fast operation. $4 \mathrm{~N} 35 \mathrm{~s}$ are commonly used in interfacing an electronic circuit with the parallel port of a computer.

\section{ULN2003}

ULN2003 is a high voltage and high current Darlington array IC. It contains seven open collector Darlington pairs with common emitters. A darling ton pair is an arrangement of two bipolar transistors. ULN2003 belongs to the family of ULN200X series of ICs. ULN2003 is for 5V TTL, CMOS logic devices. These ICs are used when driving a wide range of loads and are used as relay drivers, display drivers, line drivers etc. ULN2003 is also commonly used while driving Stepper Motor. Each channel or Darlington pair in ULN2003 is rated at $500 \mathrm{~mA}$ and can withstand peak current of $600 \mathrm{~mA}$. The inputs and outputs are provided opposite to each other in the pin layout. It is used for switching electricity connection.

\section{LCD}

LCD (Liquid Crystal Display) is the technology used for displays in notebook and other smaller computers. Like lightemitting diode (LED) and gas-plasma technologies, LCDs allow displays to be much thinner than cathode ray tube (CRT) technology. It displays the Energy Meter reading units and balance.

B. Web Server Interface Design

The pulse for every unit from the energy meter is monitored using Arduino controller. The read_pulse() is used for reading the pulse from energy meter through the optocoupler IC and updates the unit and balance. For every 30 seconds the Arduino controller tries to send the value received to the central public server through IoT using GSM modem.

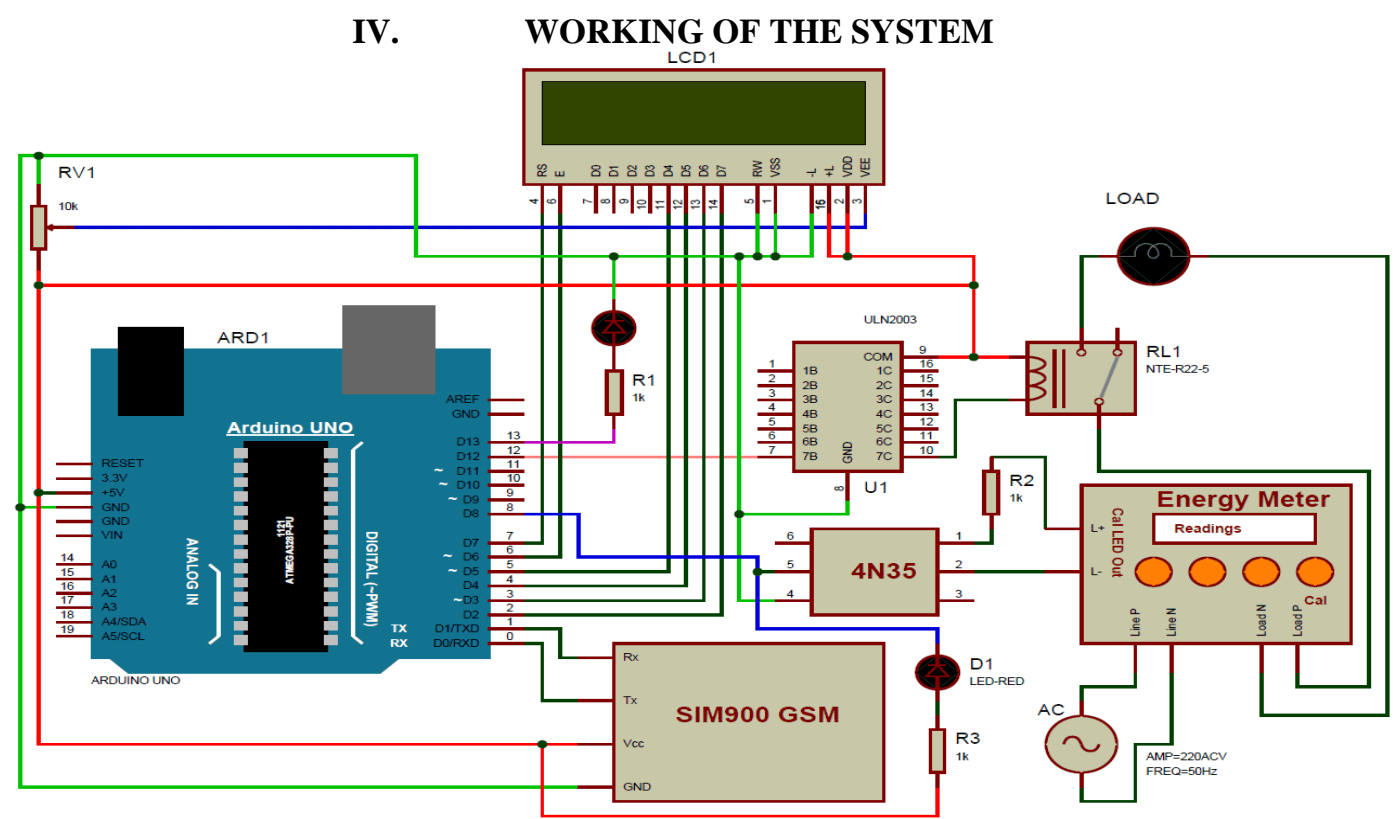

Fig. 4 Circuit Diagram 
Initially we interface Energy Meter with Arduino using the pulse LED (calibration or CAL). This CAL LED is connected to Arduino through an optocoupler IC. Depending on how many times the pulse of the LED blinks in a minute the readings are recorded by the Arduino. The formula used to calculate the pulse is given as:

Pulse $=($ pulse $/$ rate $*$ watt $*$ time $) /(1000 * 3600)$

So pulses for $100 \mathrm{~W}$ bulb in 60 seconds with the energy meter of $3200 \mathrm{imp} / \mathrm{kwh}$, the pulse rate can be calculated as:

Pulse $=(3200 * 100 * 60) /(1000 * 3600)=5.33$ pulse $/ \mathrm{min}$

When we power up the system it reads the previous values of rupees stored EEPROM and restores them into variables. Then it checks the available balance with predefined values and takes action according to them. After this we initialize LCD, serial communication and GSM in the Arduino program. Using the GPRS shield Suli_master which leads to SIM 900_serial_debug command used for serial communication. gsm_init() function is used for initializing the GSM module to operate with the system. SIM 900 accepts only AT commands. In check_status function system reads connection and balance condition, like whether electricity balance is greater than the defined limit. If the balance is less than set value, it alerts the user by sending a notification of low balance on the account as well as a SMS on the registered number. If balance is lower than the defined limit for a set period of time electricity is cut immediately using relay driver. Internet of things (IoT) is the main method of communication between Energy meter and the Web Server. It is ideally suitable for data transfer over an always online connection between a central location and mobile devices.

The reading information from the energy meter is uploaded to a central database via IoT through the GSM modem. The following is the flow of functionality for SIM 900:

- The modem is instructed first by the Arduino controller to establish a connection to the gateway and receive an IP address on the internet.

- Once the modem obtains its IP address it initiates a connection to the Web Server.

- When the connection handshake is finished all the data from Arduino would be uploaded to the web Server through the modem.

- When the data is finally sent, the modem again relinquishes the Transmission Control Protocol (TCP) connection from the server and waits for new data to establish the connection again.

Further we use application programming interface (API) set of subroutine definitions, protocols, and tools for building application software. In general terms, it is a set of clearly defined methods of communication between various software devices. It with all types of communication protocols. An API has a particular set of triggers and actions. Data available to the user will be in the form of an email trigger which would consist of the low balance notification which will alert the user to recharge immediately.

\section{CONCLUSION}

The design of IoT based prepaid electricity makes the user pay for their electricity usage before consuming it. This system saves the consumer's time by making them work leaner. The calculation of power cost is simple and doesn't involve delays. Thefts can be avoided by this secure way of central database server. An arrangement is also made to intimate the user with the help of GSM communication module when their credit balance goes low. It has become possible to record live readings due to the implementation of Arduino controller. This project can therefore enlighten time management, unnecessary trips, book-keeping and billing because it gives an accurate account of units driven.

\section{FUTURE SCOPE}

The tedious task of appointing members to calculate the distribution and consumption of electricity meters in every region can be eliminated as the bills are generated automatically. No callous amounts of electricity would be lost thereby saving energy for interior rural networks which face a lack of electricity. Theft of electricity can be avoided by tamper proof energy meters. Also the errors in the closed system can be identified quickly. System can be improved by using newer methods such as Raspberry-pi instead of Arduino to make it faster.

\section{REFERENCES}

1. YujunBao and Xiaoyan Jiang, "Design of electric Energy Meter for long-distance data information transfers which based upon GPRS", ISA2009. International Workshop on Intelligent Systems and Applications, 2009. 2

2. H.G.Rodney Tan,C.H. Lee,V.H.Mok, “Automatic power meter reading system using GSM network", The 8 Conference (IPEC 2007). International Power Engineering

3. Bharath P, Ananth N, Vijetha S, Jyothi Prakash K. V. "Wireless automated digital Energy Meter", ICSET 2008.

4. Subhashis Maitra, "Embedded Energy Meter- A new concept to measure the energy consumed by a consumer and to pay the bill", Power System Technology and IEEE Power India Conference, 2008. 